\title{
A local regularity of the complex Monge-Ampère equation
}

\author{
Zbigniew Błocki · Sławomir Dinew
}

Received: 6 May 2010 / Revised: 31 August 2010 / Published online: 23 November 2010

(C) The Author(s) 2010. This article is published with open access at Springerlink.com

\begin{abstract}
We prove a local regularity (and a corresponding a priori estimate) for plurisubharmonic solutions of the nondegenerate complex Monge-Ampère equation assuming that their $W^{2, p}$-norm is under control for some $p>n(n-1)$. This condition is optimal. We use in particular some methods developed by Trudinger and an estimate for the complex Monge-Ampère equation due to Kołodziej.
\end{abstract}

\section{Introduction}

The aim of this note is to prove the following a priori estimate for the complex Monge-Ampère equation:

Theorem Assume that $p>n(n-1)$. Let $u \in W^{2, p}(\Omega)$ (that is partial derivatives of $u$ up to the second order are in $L^{p}(\Omega)$ ), where $\Omega$ is a domain in $\mathbb{C}^{n}$, be a plurisubharmonic solution of

$$
\operatorname{det}\left(u_{z_{j} \bar{z}_{k}}\right)=\psi>0
$$

Assume that $\psi \in C^{1,1}(\Omega)$ (that is $\psi \in C^{1}(\Omega)$ and the second partial derivatives of $\psi$ are Lipschitz continuous). Then for $\Omega^{\prime} \Subset \Omega$ we have

Partially supported by the projects N N201 268335 and 189/6 PR EU/2007/7 of the Polish Ministry of Science and Higher Education.

Z. Błocki $(\varangle) \cdot$ S. Dinew

Institute of Mathematics, Jagiellonian University, Łojasiewicza 6, 30-348 Kraków, Poland

e-mail: Zbigniew.Blocki@im.uj.edu.pl

S. Dinew

e-mail: Slawomir.Dinew@im.uj.edu.pl 


$$
\sup _{\Omega^{\prime}} \Delta u \leq C,
$$

where $C$ is a constant depending only on $n, p, \operatorname{dist}\left(\Omega^{\prime}, \partial \Omega\right), \inf _{\Omega} \psi,\|\psi\|_{C^{1,1}(\Omega)}$ and $\|\Delta u\|_{L^{p}(\Omega)}$.

By a complex version of the Evans-Krylov theory (see e.g. [5] or [11]), once one has an upper bound for the Laplacian (and thus for mixed complex second derivatives) then also a $C^{2, \alpha}$-estimate follows. We thus get the following local regularity of plurisubharmonic solutions of (1)

$$
u \in W_{l o c}^{2, p} \text { for some } p>n(n-1), \quad \psi \in C^{\infty} \Longrightarrow u \in C^{\infty}
$$

For $p>2 n(n-1)$ this (and the theorem) is a consequence of a general real theory from [13] (see [4]). For $p>n^{2}$ a similar a priori estimate for $C^{3}$-solutions (without a regularity result though) was recently shown in [7].

The main point about our result is that the condition $p>n(n-1)$ is essentially optimal. The fact that it is false for $p<n(n-1)$ follows from a complex counterpart of Pogorelov's example [10] from [4]: the function

$$
u(z)=\left(1+\left|z_{1}\right|^{2}\right)\left|z^{\prime}\right|^{2-2 / n},
$$

where $z^{\prime}=\left(z_{2}, \ldots, z_{n}\right)$, is in $W_{l o c}^{2, p}$ if and only if $p<n(n-1)$, plurisubharmonic in $\mathbb{C}^{n}$, and satisfies

$$
\operatorname{det}\left(u_{z_{j} \bar{z}_{k}}\right)=c_{n}\left(1+\left|z_{1}\right|^{2}\right)^{n-2} \in C^{\infty}\left(\mathbb{C}^{n}\right)
$$

( $c_{n}$ is a constant depending only on $n$ ) in the weak sense of [2].

The corresponding estimates and regularity for the real Monge-Ampère equation can be found in [14].

The main tool in the proof of Theorem will be the following estimate of Kołodziej [8] (see also [9]): if a plurisubharmonic $u$ with $u \geq 0$ on $\partial \Omega$ solves (1) (with $\psi$ satisfying only $\psi \geq 0$ ) then for $q>1$ we have

$$
\sup _{\Omega}(-u) \leq C(q, n, \operatorname{diam} \Omega)\|\psi\|_{L^{q}(\Omega)}^{1 / n}
$$

This result for $q=2$ is due to Cheng and Yau (see $[1,6]$ ).

\section{Proof of Theorem}

By $C_{1}, C_{2}, \ldots$ we will denote possibly different positive constants depending only on the required quantities. Without loss of generality we may assume that $\Omega=B$ is the unit ball in $\mathbb{C}^{n}$ and that $u$ is defined in some neighborhood of $\bar{B}$. We will use the notation $u_{j}=u_{z_{j}}, u_{\bar{j}}=u_{\bar{z}_{j}}$ and $\Delta u=\sum_{j} u_{j} \bar{j}$. As usual, by $\left(u^{i} \bar{j}\right)$ we will denote the inverse transposed of $\left(u_{i} \bar{j}\right)$. 
We will first prove Theorem assuming that $u$ is in $C^{4}$. Differentiating (1) w.r.t. $z_{p}$ and $\bar{z}_{p}$ we will get

$$
u^{i} \bar{j}_{i \bar{j} p}=(\log \psi)_{p}
$$

and

$$
u^{i \bar{j}} u_{i \bar{j} p \bar{p}}=(\log \psi)_{p \bar{p}}+u^{i \bar{l}} u^{k \bar{j}} u_{k \bar{l} \bar{p}} u_{i \bar{j} p}
$$

Therefore

$$
u^{i \bar{j}}(\Delta u)_{i \bar{j}} \geq \Delta(\log \psi)
$$

We will now use an idea from [12]. For some $\alpha, \beta \geq 2$ to be determined later set

$$
w:=\eta(\Delta u)^{\alpha}
$$

where

$$
\eta(z):=\left(1-|z|^{2}\right)^{\beta}
$$

Then

$$
w_{i}=\eta_{i}(\Delta u)^{\alpha}+\alpha \eta(\Delta u)^{\alpha-1}(\Delta u)_{i}
$$

and

$$
\begin{aligned}
u^{i \bar{j}} w_{i \bar{j}}= & \alpha \eta(\Delta u)^{\alpha-1} u^{i \bar{j}}(\Delta u)_{i \bar{j}}+\alpha(\alpha-1) \eta(\Delta u)^{\alpha-2} u^{i \bar{j}}(\Delta u)_{i}(\Delta u)_{\bar{j}} \\
& +2 \alpha(\Delta u)^{\alpha-1} \operatorname{Re}\left(u^{i \bar{j}} \eta_{i}(\Delta u)_{\bar{j}}\right)+(\Delta u)^{\alpha} u^{i \bar{j}} \eta_{i \bar{j}}
\end{aligned}
$$

By (4) and the Schwarz inequality for $t>0$

$$
\begin{aligned}
u^{i \bar{j}} w_{i \bar{j}} \geq & \alpha \eta(\Delta u)^{\alpha-1} \Delta(\log \psi)+\alpha(\alpha-1) \eta(\Delta u)^{\alpha-2} u^{i \bar{j}}(\Delta u)_{i}(\Delta u)_{\bar{j}} \\
& -t \alpha(\Delta u)^{\alpha-1} u^{i \bar{j}}(\Delta u)_{i}(\Delta u)_{\bar{j}}-\frac{1}{t} \alpha(\Delta u)^{\alpha-1} u^{i \bar{j}} \eta_{i} \eta_{\bar{j}}+(\Delta u)^{\alpha} u^{i \bar{j}} \eta_{i \bar{j}}
\end{aligned}
$$

Therefore with $t=(\alpha-1) \eta / \Delta u$ we get

$$
u^{i \bar{j}} w_{i \bar{j}} \geq \alpha \eta(\Delta u)^{\alpha-1} \Delta(\log \psi)+(\Delta u)^{\alpha} u^{i \bar{j}}\left(\eta_{i \bar{j}}-\frac{\alpha}{\alpha-1} \frac{\eta_{i} \eta_{\bar{j}}}{\eta}\right) .
$$

We now have

$$
\begin{aligned}
\eta_{i} & =-\beta z_{i} \eta^{1-1 / \beta} \\
\eta_{i} \bar{j} & =-\beta \delta_{i} \eta^{1-1 / \beta}+\beta(\beta-1) \bar{z}_{i} z_{j} \eta^{1-2 / \beta}
\end{aligned}
$$


and thus

$$
\left|\eta_{i \bar{j}}\right|, \quad\left|\frac{\eta_{i} \eta_{\bar{j}}}{\eta}\right| \leq C(\beta) \eta^{1-2 / \beta}
$$

We will get

$$
u^{i \bar{j}} w_{i \bar{j}} \geq-C_{1}(\Delta u)^{\alpha-1}-C_{2} w^{1-2 / \beta}(\Delta u)^{2 \alpha / \beta} \sum_{i, j}\left|u^{i \bar{j}}\right| .
$$

Fix $q$ with $1<q<p /(n(n-1))$. Since $\|\Delta u\|_{p}$ (this way we will denote norms in $\left.L^{p}(B)\right)$ is under control, it follows that $\left\|u_{i} \bar{j}\right\|_{p}$ and $\| u^{i} \bar{j}_{p /(n-1)}$ are as well. It follows that for

$$
\alpha=1+\frac{p}{q n}, \quad \beta=2\left(1+\frac{q n}{p}\right)
$$

we have

$$
\left\|\left(u^{i \bar{j}} w_{i \bar{j}}\right)_{-}\right\|_{q n} \leq C_{3}\left(1+\left(\sup _{B} w\right)^{1-2 / \beta}\right),
$$

where $f_{-}:=-\min (f, 0)$.

By [2] we can find continuous plurisubharmonic $v$ vanishing on $\partial B$ and such that

$$
\operatorname{det}\left(v_{i \bar{j}}\right)=\left(\left(u^{i \bar{j}} w_{i \bar{j}}\right)_{-}\right)^{n}
$$

(weakly). Essentially by an inequality between arithmetic and geometric means (see [3] how to extend it to the weak case) we have

$$
\begin{aligned}
u^{i \bar{j}} v_{i \bar{j}} & \geq n\left(\operatorname{det}\left(u^{i \bar{j}}\right)\right)^{1 / n}\left(\operatorname{det}\left(v_{i \bar{j}}\right)\right)^{1 / n} \\
& =n \psi^{-1 / n}\left(u^{i \bar{j}} w_{i \bar{j}}\right)_{-} \\
& \geq-\frac{1}{C_{4}} u^{i \bar{j}} w_{i \bar{j}}
\end{aligned}
$$

It follows that $w \leq-C_{4} v$ and by Kołodziej's inequality (3)

$$
\begin{aligned}
\sup _{B} w & \leq C_{5}\left\|\operatorname{det}\left(v_{i \bar{j}}\right)\right\|_{q}^{1 / n} \\
& =C_{5}\left\|\left(u^{i \bar{j}} w_{i \bar{j}}\right)_{-}\right\|_{q n} \\
& \leq C_{6}\left(1+\left(\sup _{B} w\right)^{1-2 / \beta}\right) .
\end{aligned}
$$

Therefore $w \leq C_{7}$ and the desired estimate follows if $u \in C^{4}$. 
Now assume that the solution is just in $W^{2, p}$. Similarly to [2], instead of $\Delta u$ we will consider for $\varepsilon>0$ the following approximations to the Laplacian

$$
T=T_{\varepsilon} u=\frac{n+1}{\varepsilon^{2}}\left(u_{\varepsilon}-u\right),
$$

where

$$
u_{\varepsilon}(z)=\frac{1}{\lambda(B(z, \varepsilon))} \int_{B(z, \varepsilon)} u \mathrm{~d} \lambda
$$

and $\lambda$ denotes the Lebesgue measure in $\mathbb{C}^{n}$. Since $T_{\varepsilon} u \rightarrow \Delta u$ weakly as $\varepsilon \rightarrow 0$, it is enough to show a uniform upper bound for $T$ independent of $\varepsilon$.

By [2] we have

$$
u^{i \bar{j}} u_{\varepsilon, i \bar{j}} \geq n \psi^{-1 / n}\left(\operatorname{det}\left(u_{\varepsilon, i}\right)\right)^{1 / n} \geq n \psi^{-1 / n}\left(\psi^{1 / n}\right)_{\varepsilon}
$$

and thus, coupling this with $u^{i} \bar{j} u_{i \bar{j}}=n$, we obtain the following counterpart of (4)

$$
u^{i \bar{j}} T_{i \bar{j}} \geq n \psi^{-1 / n} T_{\varepsilon}\left(\psi^{1 / n}\right) \geq-C_{8}
$$

Changing the definition of $w$ to $\eta T^{\alpha}$ (since $u$ is plurisubharmonic, $T$ is nonnegative, hence $T^{\alpha}$ is well defined) and repeating the previous computations we will get

$$
u^{i \bar{j}} w_{i \bar{j}} \geq C_{9} T^{\alpha-1}-C_{10} w^{1-2 / \beta} T^{2 \alpha / \beta} \sum_{i, j}\left|u^{i \bar{j}}\right|
$$

The rest of the proof is now the same as before.

Acknowledgments Part of the research was done while the second named author was visiting the Princeton University. He would like to thank this institution for the perfect working conditions and hospitality and especially professor Gang Tian for his encouragement and help.

Open Access This article is distributed under the terms of the Creative Commons Attribution Noncommercial License which permits any noncommercial use, distribution, and reproduction in any medium, provided the original author(s) and source are credited.

\section{References}

1. Bedford, E.: Survey of pluri-potential theory. In: Fornæss, J.E. (ed.) Several Complex Variables. Proceedings of the Mittag-Leffler Institute, vol. 1987-1988. Princeton University Press, New Jersy (1993)

2. Bedford, E., Taylor, B.A.: The Dirichlet problem for a complex Monge-Ampère equation. Invent. Math. 37, 1-44 (1976)

3. Błocki, Z.: The complex Monge-Ampère operator in hyperconvex domains. Ann. Scuola Norm. Sup. Pisa 23, 721-747 (1996)

4. Błocki, Z.: On the regularity of the complex Monge-Ampère operator. In: Complex Geometric Analysis in Pohang, 1997. Contemp. Math., vol. 222, pp. 181-189. Amer. Math. Soc., Providence (1999) 
5. Błocki, Z.: Interior regularity of the complex Monge-Ampère equation in convex domains. Duke Math. J. 105, 167-181 (2000)

6. Cegrell, U., Persson, L.: The Dirichlet problem for the complex Monge-Ampère operator: stability in $L^{2}$. Michigan Math. J. 39, 145-151 (1992)

7. He, W.: On regularity of complex Monge-Ampère equations. arXiv:1002.4825v2

8. Kołodziej, S.: Some sufficient conditions for solvability of the Dirichlet problem for the complex Monge-Ampère operator. Ann. Pol. Math. 65, 11-21 (1996)

9. Kołodziej, S.: The complex Monge-Ampère equation. Acta Math. 180, 69-117 (1998)

10. Pogorelov, A.V.: The Dirichlet problem for the multidimensional analogue of the Monge-Ampère equation. Dokl. Akad. Nauk SSSR 201, 790-793 (1971) [transl.: Soviet Math. Dokl. 12, 1727-1731 (1971)]

11. Siu, Y.-T.: Lectures on Hermitian-Einstein metrics for stable bundles and Kähler-Einstein metrics. Birkhäuser, Basel (1987)

12. Trudinger, N.S.: Local estimates for subsolutions and supersolutions of general second order elliptic quasilinear equations. Invent. Math. 61, 67-79 (1980)

13. Trudinger, N.S.: Regularity of solutions of fully nonlinear elliptic equations. Boll. Un. Mat. Ital. A (6) 3, 421-430 (1984)

14. Urbas, J.: Regularity of generalized solutions of Monge-Ampère equations. Math. Z. 197, 365393 (1988) 